\title{
FOSCOLO TRA LE MASCHERE: SIMULAZIONE E DISSIMULAZIONE COME STRUMENTI DI RIFLESSIONE SULL'IDENTITÀ NELL'OPERA DI UGO FOSCOLO
}

\author{
Angela Alexandra D’ORSO \\ Università Carolina, Praga
}

\begin{abstract}
En): The article deals with Foscolo's contribution to autobiographic writing. The study focuses on methods that the author uses, having a particular attention for the foscolanian masks and their functions. The analysis of Ultime lettere di Jacopo Ortis, Sesto Tomo dell' io and Notizia intorno a Didimo Chierico makes us declare that Foscolo continued, throughout his literary career, a reflection on the concept of identity which had the practice of simulation in the instrument of knowledge.
\end{abstract}

Keywords (En): dissimulation ; simulation ; novel ; autobiography ; self-portrayal ; mask

Parole chiave (It): dissimulatio ; simulatio ; romanzo ; autorappresentazione ; maschera

\section{Introduzione}

Oserei definire, la civiltà: la perfetta [arte] di fingere.

E la virtù: il secreto di mascherare tutti i volti.

(Ugo Foscolo, Frammenti di un romanzo autobiografico)

Nel 1988 Carlo Dionisotti pubblica i suoi Appunti sui moderni, una raccolta di discorsi su autori del Sette e Ottocento tra i quali compare, prima di Leopardi e Manzoni, il nome di Ugo Foscolo. La modernità di Foscolo è legata al particolare momento storico in cui visse: la rivoluzione politica e militare del 1796-97 non produsse forse alcuna nuova dottrina letteraria ma permise al Foscolo «benché non avesse un nome, né un'arte, né mezzi di fortuna [...] di disporre della sua vocazione letteraria liberamente, gettandosi allo sbaraglio, con la testa alta, in una società d'improvviso aperta [...] a feroci petti e altissimi ingegni, quale che fosse la loro origine.» (DIONISOTTI, 1988: 35).

È in questo contesto che, nel 1798, il primo romanzo moderno in lingua italiana vede la luce; la modernità delle Ultime lettere di Jacopo Ortis non risiede però esclusivamente nella struttura epistolare o nella componente introspettiva, a rendere innovativa la narrazione è il legame tra Foscolo e il protagonista del suo romanzo:

L'esule italo-greco faceva spicco per la sua eccezionale personificazione di due lingue e letterature che in quel momento erano predilette entrambe dagli Inglesi. [...] Avrebbe potuto semplicemente insegnare le due lingue [...] Ma Jacopo Ortis non poteva sopravvivere in figura di pedagogo. Mancò al Foscolo [...] la forza di smentire la propria anacronistica identificazione col personaggio del romanzo e di accettare la realtà di un esilio diverso. (DiONISOTTI, 1988:76)

La sovrapposizione Foscolo - Ortis a cui accenna Dionisotti estende la questione della modernità al genere dell'autobiografia. Jacopo Ortis, personaggio 
sospeso tra realtà e finzione, è l'emblema del complicato rapporto che Foscolo intrattenne, in tutto il corso della sua carriera, con il genere letterario.

Che si tratti di una relazione speciale è possibile evincerlo dall'assenza, nella bibliografia foscoliana, di un'autobiografia esplicita ${ }^{1}$ a cui si sostituisce, invece, una serie di scritti autobiografici in cui l'autore simula, dissimula, finge di essere un altro.

Quella di Jacopo non è infatti l'unica maschera foscoliana; è possibile individuare almeno altri due camuffamenti : Il Lorenzo del Sesto tomo dell'io e Didimo Chierico, personaggi la cui interpretazione rimane ancora problematica.

Scopo principale del presente lavoro è indagare, senza necessariamente voler giungere ad una soluzione definitiva, il contributo di Foscolo al genere dell'autobiografia. I travestimenti foscoliani sono funzionali ad una riflessione sul concetto di identità o sono un semplice gioco letterario? Possono essere considerati l'unica soluzione possibile per un autore ossessionato dall'autorappresentazione oppure costituiscono il preambolo di un'autobiografia progettata ma mai redatta?

\section{1. «Pronto, iracondo, inquïeto, tenace»: l'ossessione per l'autorappre- sentazione nell'opera di Ugo Foscolo come premessa all'autobiografismo}

Se parlare di autobiografia foscoliana non è lecito in quanto manca tra le opere dell'autore un testo che appartenga dichiaratamente al genere, credo sia possibile riconoscere nella sua produzione una spiccata tendenza all'autorappresentazione. L'immaginario collettivo foscoliano si avvale di un apparato di raffigurazioni decisamente ampio. Pochi scrittori coevi godettero, di fatto, di una fortuna iconografica pari a quella del poeta. Questo dato - che dimostra, se non altro, l'accondiscendenza dell'autore alla diffusione del proprio ritratto - supporta e giustifica la marcata disposizione all' autobiografismo nell'opera di Foscolo.

Nella prosa l'autonarrazione è esplicita e cospicua: è possibile imbattersi, fin dalle prime pubblicazioni, nelle tracce di un ritratto chiaro ed incisivo del poeta:

Giovane qual mi son io, nato in Grecia, educato fra Dalmati, e balbettante da soli quattr'anni in Italia, né dovea, né poteva cantare ad uomini liberi ed Italiani. Ma l'alto genio di Libertà che m'infiamma e che mi rende Uomo, libero, e Cittadino di patria non in sorte toccata, ma eletta, mi dà i diritti dell'Italiano. (Foscolo, 1961: 331)

Sono queste le parole che nel 1797 Foscolo sceglie per raccontare le proprie coordinate anagrafiche alla città di Reggio, poche righe ma densissime di significato, in grado di descrivere efficacemente la condizione interculturale ed interlinguistica che caratterizzò la sua vita.

\footnotetext{
1 Se si esclude un piccolo frammento autobiografico in francese presumibilmente del 1814, pubblicato da Trevisan in Ugo Foscolo e la sua fede politica, Mantova, 1872.
} 
Altro momento centrale della narrazione foscoliana è l'Orazione pavese che introduce i saggi Dell'Origine e dell'Ufficio della Letteratura. L'orazione ospita una riflessione sull'esilio di Dante nella quale è evidente l'accentuazione da parte di Foscolo delle avversità che contraddistinguono la biografia dantesca. Ebbene, questa componente emerge come tratto tipico della propria caratterizzazione sia in prosa che in poesia: riferendosi a se stesso, Foscolo si descrive come un intellettuale controcorrente e solitario nelle proprie battaglie, un ritratto che evoca vagamente quello di Dante appena citato, poeta rifiutato e esiliato:

Sempre il Foscolo è vissuto e ha scritto in guerra aperta con gli autori suoi contemporanei e con i partiti politici trionfanti [...]. (Foscolo, 2005: 148)

È forse da ritenere ulteriore manifestazione di questa propensione la stesura della Lettera apologetica - composta da Foscolo appena due anni prima della morte - attraverso la quale mira a modellare un proprio ritratto apologetico, ponendosi come un eroe plutarchesco mosso da sentimenti puri e assoluti, ingiustamente perseguitato dai letterati italiani asserviti al potere.

$\mathrm{Al}$ carteggio dell'autore appartiene invece un autoritratto fisico ${ }^{2}$ indirizzato ad un amico nel 1795 che in poesia corrisponde parzialmente ad un componimento della Raccolta Naranzi, un codicetto oggi perduto in cui l'autore collezionò le sue prime scritture in rima:

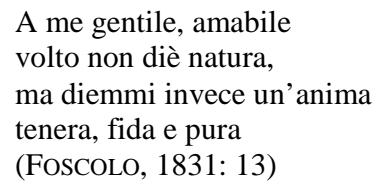

Il ritratto prenderà piena forma lirica con il sonetto Solcata ho la fronte. Apparso per la prima volta nell'Edizione delle Rime del 1802, l'ultima redazione risale al 1827, appena quattro mesi prima della morte del poeta:

Solcata ho la fronte; occhi aggrottati, intenti;

Crin fulvo, emunte guance, ardito aspetto;

Labbri tumidi arguti al riso lenti;

Capo chino, bel collo, irsuto petto;

Membra esatte, vestir semplice eletto;

Ratti i passi i pensier gli atti gli accenti:

Prodigo, sobrio, umano, ispido, schietto;

Avverso al Mondo, avversi a me gli eventi.

Solo i più giorni e mesto e ognor pensoso,

Alle speranze incredulo e al timore,

Il pudor mi fa vile e prode l'ira.

\footnotetext{
2 «Di volto non bello, ma stravagante e d'un aria libera; di crini non biondi, ma rossi; di naso acquilino, ma non piccolo e non grande; d'occhi mediocri, ma vivi; di fronte ampia, di ciglia bionde e grosse, e di mento ritondo. La mia statura non è alta, ma mi si dice che deggio crescere; tutte le mie membra sono ben formate dalla natura, e tutte hanno del ritondo e del grosso. Il portamento non scuopre nobiltà nè letteratura, ma è agitato trascuratamente. Eccovi il mio ritratto» (SoFFICI; 1922: 283-284)
} 
Mi parla astuta la ragion; ma il core

Ricco di vizj e di virtù delira,

E sol da morte aspetterò riposo.

(FosCOLO, 1992: 92-93)

Se poi si considera la scelta di organizzare i sonetti come un canzoniere struttura che impone al poeta, secondo il modello petrarchesco, di ordinare il «canto dell'io» seguendo un percorso evolutivo e circolare - il liet motiv della autorappresentazione è estendibile in Foscolo anche ad un piano macrotestuale.

Un altro momento d'intenso autobiografismo è inoltre affidato al carme $L e$ Grazie, componimento in cui la riflessione sulla tradizione letteraria ha la sua espressione apicale: dopo una serie di peripezie e fughe le tre dee vagano alla ricerca di un luogo in cui stabilirsi. Ebbene, si fatica molto a non ravvisare nella migrazione delle Grazie «il percorso del poeta stesso, nato e cresciuto in Grecia [...] poi maturato nella sua nuova patria, l'Italia, e ormai senza una vera collocazione, alla ricerca di un luogo in cui fuggire [...] per elaborare una nuova forma di poesia civile, un nuovo modo per mettere l'arte al servizio della società umana». (ANTONELLI, SAPEGNO, 2008: 170)

\section{Le maschere foscoliane: Jacopo, Lorenzo, Didimo Chierico}

\subsection{Le Ultime lettere di Jacopo Ortis}

L'ossessione per la narrazione del sé che emerge da prosa e poesia sembra assumere i tratti dell'autobiografismo all'interno del romanzo Ultime lettere di Jacopo Ortis.

Sono numerose infatti le corrispondenze tra Jacopo e Foscolo: la scelta di descrivere il protagonista come un giovane uomo in fuga da chi detiene il potere, come lettore appassionato di Plutarco; la collocazione storico-geografica degli eventi, la determinante presenza del personaggio di Parini.

All'interno dell'Ortis traspare, chiaramente, la «grecità» di Foscolo: una pagina del romanzo, aggiunta all'edizione del 1816, fa riferimento all'usanza di origine greca che prevede per la donna la recisione di una ciocca di capelli in segno di lutto per la morte dell'amato rappresentato, in questo caso, da Jacopo, metaforicamente morto per Teresa dopo il matrimonio con Edoardo:

fra il cristallo e la scanellatura di dentro della cornice [Jacopo] trovò una lunga treccia di capelli che Teresa, alcuni giorni prima delle nozze, s'era tagliati senza che veruno il sapesse. (Foscolo 1993: 144)

Un altro elemento autobiografico è riconoscibile nella benedizione richiesta da Jacopo alla madre:

Sua madre gli si approssimò e con volto rassegnato [...] gli disse: [...] Vivi, io scelgo di piangere senza di te piuttosto che vederti - imprigionato - e morto. [...] Jacopo le strinse la mano e le chiese la sua benedizione. Ed ella alzando le palme: Ti benedico - Ti benedico; e piaccia anche a Dio Onnipotente di benedirti. (Foscolo, 1993: p. 144) 
Saremmo di fronte al calco di una tradizione greco-ortodossa a cui Foscolo fa riferimento nelle lettere inviate alla madre:

Or addio, addio, Addio Pippì; ama tua madre, e ascolta con religione i sentimenti che t'ispira. Cara Rubina, ti mando un bacio - e mille baci a te, Madre mia, di cui chiedo tutte le sere che vado a letto la santa benedizione [...]. (Foscolo, 1853: 107)

Anche a livello prettamente testuale è possibile individuare delle equivalenze. Già Goffis ${ }^{3}$ ha scritto a proposito dell'utilizzo da parte di Foscolo di materiale epistolare privato all'interno dell'Ortis. Mi riferisco in particolare a quattordici lettere indirizzate fra l'autunno 1813 e i primi mesi del 1814 a Lucietta Frapolli.

Altrettanto interessanti sono le porzioni di testo in cui Jacopo chiede insistentemente a Teresa di inviargli un suo ritratto:

Perdonami Teresa, io ho funestato la tua giovinezza [...] Mandami in qualunque tempo, in qualunque luogo il tuo ritratto. Se l'amicizia, se l'amore - o la compassione e la gratitudine ti parlano ancora per questo sconsolato - non negarmi il ristoro che addolcirà tutti i miei patimenti. (FosCOLO 1993: 80)

Per carità non mi negare il tuo ritratto. Consegnalo a Lorenzo: e s'ei non me lo potrà far arrivare, lo custodirà come eredità santa, che gli ricorderà sempre le tue virtù, e la tua bellezza. (FosCOLO 1993: 81)

Tale richiesta, articolata con modalità simili e altrettanta ostinazione da Foscolo, è presente in più punti del carteggio privato con Antonietta Fagnani Arese, sua amante dal 1801 al 1803:

Addio intanto. Ti domando per l'ultima volta il tuo ritratto, non perch'io creda d'essere esaudito, ma per obbedire al mio cuore. [...] Addio. Addio. (Foscolo, 2008: lettera XXXI)

dammi il tuo ritratto, il tuo solo ritratto; io me ne andrò in campagna, a Venezia.... dove mi strascinerà il mio destino; ti lascerò tranquilla e libera. Porterò con me la mia tremenda passione, le tue lettere, tutte le tristi e care memorie del tuo amore (FosCOLO, 2008: lettera XXXV)

Almeno tu hai avuta la cura di farmi avere il tuo ritratto; unico conforto che mi possa rimanere [...]. (FosCOLO, 2008: lettera LII)

Il ritratto avrebbe dunque, sia per Foscolo che per Jacopo, una funzione consolatoria, al punto da rappresentare una sorta di vicario dell'amata stessa. La sostituzione appare tragicamente lampante nelle ultime pagine dell'Ortis:

Fa ch'io sia sepolto, così come sarò trovato, in un sito abbandonato, di notte senza esequie, senza lapide, sotto i pini del colle che guarda la chiesa. Il ritratto di Teresa sia sotterrato col mio cadavere. (FosCOLO, 1993: 150)

\footnotetext{
${ }^{3}$ Per un approfondimento sull'impiego del carteggio Foscolo - Frapolli rimandiamo a GoFFIS Cesare (1957), Ortis non scritto del 1814 in «Nuova Antologia» XCII 1957, pp. 53-84.
} 
La pagina in questione sembra esasperare e riadattare un motivo presente anche nello scambio epistolare con Antonietta:

sì, odilo, t'amo; odilo mille volte. [...] e sceglierei piuttosto di unire il mio cadavere al tuo, anziché sopravviverti glorioso e felice. (FosCOLO, 2008: lettera XVI).

Le corrispondenze tra Foscolo e Jacopo sono dunque numerose ed interessano sia il piano testuale che quello macrotestuale dell'opera. Credo non si possa negare, dunque, che si tratti di un romanzo autobiografico.

Tuttavia, considerare le somiglianze individuate come frutto unicamente di autobiografismo rischia di essere, a mio parere, riduttivo: Jacopo è Foscolo nella misura in cui l'autore gli affida la sua anima più romantica, quella che guida il protagonista fino al gesto estremo che Foscolo, di fatto, non compì mai.

\subsection{Il Sesto tomo dell'io o Frammenti di un romanzo autobiografico}

Il Sesto tomo dell'io, romanzo meno noto e mai concluso, condivide con l'Ortis il rimaneggiamento del carteggio privato. Il Sesto tomo o Frammenti di un romanzo autobiografico, titolo che alcuni editori hanno preferito, è costituito da una ventina di fogli autografi che contengono frammenti in prosa di varia natura e estensione, probabilmente redatti da Foscolo in un arco di tempo compreso tra il 1799 e il 1801, tra il primo ed il secondo Ortis. Protagonista dell'abbozzo e narratore in prima persona è Lorenzo, il quale afferma di raccontare i fatti relativi al suo ventitreesimo anno di vita. Il giovane uomo si presenta con toni decisamente più placidi rispetto allo Jacopo dell'Ortis. Alcuni dati biografici sono deliberatamente alterati rispetto alla vita di Foscolo - la scelta del nome, la data di nascita - mentre altri sembrano corrispondere completamente: le origini greche, i continui spostamenti sul territorio italiano, gli anni trascorsi in Francia:

[...] io nacqui in Grecia, [...] la giovinezza su e giù per l'Italia; la prima virilità in Francia. [...] Mio padre mi lasciò erede del suo genio ambulatorio (FosCOLO, 1890:61)

Il dato più interessante, però, riguarda un probabile rimaneggiamento del materiale appartenente al carteggio privato di Foscolo.

L'abbozzo ospita un'epistola indirizzata a Psiche che ricorda passi delle lettere all'amica Antonietta:

[...] il timore di perderti mi fa desiderare che la vita in quel sacro momento si spenga in noi insensibilmente, e che un sepolcro ci serbi congiunti per sempre... - Lascia lascia ch'io t'allontani da questi pensieri... perché devo io amareggiare la gioia della tua gioventù?... perché devo io spargere la mia malinconia sulla voluttà fatta per la tua anima angelica e per la tua celeste bellezza? (Foscolo, 2008: lettera X)

Nella Lettera a Psiche del Sesto Tomo dell'io puntuali riprese testuali occorrono in più punti rendendo evidente l'analogia tra l'autore e il suo personaggio. 
Buon giorno dunque. Che la tua bellezza e la tua gioventù sorridano sempre con l'aurora di questa mattina. Sempre? cielo [...] abbi pietà della mia giovinetta! Che ti dirò intanto? I miei mali?...no: la tua compassione sarebbe un balsamo, è vero, al mio povero cuore; non sarà però mai che io voglia avvelenare la pace e la voluttà fatte per la tua anima angelica, e per la tua sacra bellezza. (Foscolo, 1853: 53)

Un simile espediente riguarda anche la nominazione dell'amata: Foscolo soprannomina affettuosamente Antonietta «filosofessa»:

O mia filosofessa! tu mi hai tanto commosso ier l'altro con la tua lettera scritta fra i dolori: e veramente hai ragione: il fiore della vita si scolora cosí presto!» (Foscolo, 2008: lettera X). Allo stesso modo si comporta Lorenzo nella Lettera a Psiche: «Ma io scrivo a te, e non alla ippocondriaca filosofessa che comincia finalmente a moralizzare...[...]. (Foscolo, 1853: 54).

Non è possibile dare un giudizio sul Sesto tomo dell'io senza considerare il peso che Laurence Sterne esercitò su Foscolo. Il sesto tomo ha come modello The Life and Opinions of Tristram Shandy, Gentleman $(1760)^{4}$. La ripresa non riguarda solo il carattere autobiografico dell'opera ma l'inserimento di alcuni passi tradotti fedelmente dal romanzo di Sterne, una serie di stranezze tipografiche, un diffuso sperimentalismo delle strutture narrative. In Foscolo però l'«effetto Sterne» assume sfumature particolarmente eloquenti: l'autore racconta la propria vita ma lo fa fingendo di essere un altro. Il camuffamento - che inizia con l'Ortis e si perfeziona nel Sesto tomo dell'io - si complica ulteriormente con l'entrata in scena di Didimo Chierico.

\subsection{La Notizia intorno a Didimo Chierico}

Didimo Chierico è il personaggio che Foscolo sceglie come controfigura vera e propria. Egli appare per la prima volta nell'edizione pisana del 1813 del Viaggio sentimentale di Yorick lungo la Francia e l'Italia, traduzione di A Sentimental Journey through France and Italy di Laurence Sterne del 1768. Questa edizione presenta in appendice seconda la Notizia intorno a Didimo Chierico, che chiarisce il ruolo del bizzarro personaggio nella tradizione del romanzo. Didimo avrebbe affidato a Foscolo alcuni manoscritti tra cui la traduzione del Viaggio sentimentale. Dando vita ad un gioco di doppia finzione, Foscolo dichiara d'essere stato fedele alla traduzione consegnatagli dal chierico.

Risulta davvero difficile ritenere Didimo un semplice pseudonimo considerato che l'autore lo dota di un'identità ben precisa; Foscolo lo descrive come un personaggio assai originale, che si fa portavoce dello scetticismo e del distacco ironico dell'autore rispetto ai sogni e alle illusioni giovanili. Dunque Foscolo consegna ai lettori un'autobiografia che potremmo forse definire indiretta dal momento che l'autore racconta la vita di un personaggio fittizio che corrisponde

\footnotetext{
${ }^{4}$ Per un approfondimento sulle somiglianze tra Il Sesto tomo dell'io e Vita e opinioni di Tristram Shandy, gentiluomo rimandiamo CALVANI Alessandra (2004), Il viaggio italiano di Sterne, Firenze, Franco Cesati Editore.
} 
però a se stesso. Informazioni su Didimo sono rinvenibili, oltre che nella Notizia, anche in una confessione redatta in prima persona:

Nacqui di contadini e però crebbi d'animo semplice; erano poveri, e m'avvezzavano alla misericordia; e la madre mia rimanendosi vedova sei giorni dopo ch'io nacqui, mi alimentò del latte del suo petto e del sudore delle sue mani [...] Era ed è tuttavia il tugurio de' padri miei tra le feconde correnti dell'Adda, fiume, e la città di Milano [...] (FosCOLO, 1809: 100-103).

Se la vita di Lorenzo - così come raccontata nel Sesto tomo dell'io - ricorda molto quella di Foscolo, la biografia di Didimo se ne discosta decisamente.

La misteriosità del personaggio è rafforzata, inoltre, dal carattere enigmatico degli altri scritti che Foscolo gli attribuisce.

Come dichiarato dal poeta nella Notizia, all'alter ego appartiene anche l'Hypercalypseos Liber singularis, operetta che, fin dal titolo, esibisce una poetica dell'oscurità e del segreto. L'Hypercalypseos, pubblicata a Zurigo nel 1816 con falsa data e falso luogo di edizione, è la risposta alle polemiche scoppiate contro Foscolo in quei mesi a Milano fra gli intellettuali che si mostravano sempre più ostili nei confronti del poeta. L'invettiva si compone di diciannove capitoletti più una Clavis; il titolo dell'operetta è in greco, i capitoli e la Clavis sono invece interamente redatti in latino.

Un suggestivo ed indicativo occultamento potrebbe inoltre interessare il nome dell'alter ego ed il titolo dell'opera: «Didimo» significa «gemello», il titolo contiene una parola greca e lo schizzo riportato sul frontespizio ha, a mo' di motivo ornamentale, un motto greco ${ }^{5}$. Se si contestualizza poi la figura dell'alter ego all'interno delle teorie di traduzione foscoliane, il doppio Didimo-Foscolo potrebbe godere di un avvincente sottotesto: l'autore ci starebbe suggerendo che tradurre vuol dire doppiare se stessi, preservarsi reinventandosi. Questa interpretazione farebbe di Didimo Chierico l'applicazione concreta del precetto secondo cui «l'uomo facendosi traduttore non cessa, grazie al cielo, d'essere poeta» (FOSCOLO. 1933: 205)

\section{Conclusione}

Il romanzo Le Ultime lettere di Jacopo Ortis è il luogo in cui la predisposizione a raccontare di sé si fa autobiografismo. Tuttavia, poiché la caratterizzazione del protagonista ha un intento ben preciso, a mio parere non è possibile ridurre le somiglianze tra Jacopo e Foscolo a semplice espressione di una tendenza a fare della propria vita la fonte di ispirazione delle proprie opere. Jacopo Ortis è l'antagonista del Lorenzo del Sesto tomo dell'io e di Didimo Chierico. Se il primo è affidatario di una passione pericolosamente sfrenata per la verità, ai secondi Foscolo consegna quella saggezza scettica che fa preferire un distaccato

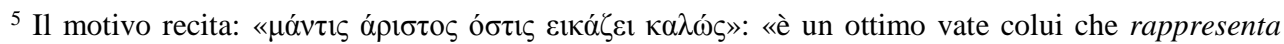
bene» (cit. Euripide in CicERONE Marco Tullio, De Divinatione II. 5). Il riferimento potrebbe prestarsi all'erasmiana interpretazione secondo cui la pratica del riprodurre - non necessariamente in maniera fedele - è strumento paideutico.
} 
approdo a ciò che è «probabile». Tutti e tre custodiscono, in egual misura, l'identità foscoliana.

La prosa - ed in particolare il romanzo - rappresenterebbe così per Foscolo un momento di riflessione sul concetto di identità; egli non medita solo sulla reale possibilità di conoscere se stessi ma anche sul ruolo dell' autorappresentazione nel riconoscimento da parte dell'altro. In questo senso l'autore è molto vicino allo smascheramento di ogni presunzione di realismo che è alla base del Tristram Shandy di Sterne.

Questa riflessione, che potrebbe essere eletta fil rouge dell'opera foscoliana, ha come strumento conoscitivo la pratica della simulazione e della dissimulazione.Se le epistole dell'Ortis non forniscono abbastanza elementi per poter parlare di autobiografia dissimulata, le pagine del Sesto tomo dell'io sembrano ospitare l'autobiografia di Foscolo che finge, però, di essere Lorenzo.

Con Didimo Chierico il gioco si complica ulteriormente: se da una parte l'autore dissimula la propria identità - Foscolo è il traduttore di Sterne ma dice che a tradurre è Didimo Chierico - dall'altra la falsificazione trasforma la biografia di Didimo Chierico nell' autobiografia di Foscolo; tuttavia la vita di Didimo, così come emerge dalla sua confessione, si discosta notevolmente dalle vicende biografiche di Foscolo.

Se è lecito pensare che simulazione e dissimulazione rappresentino uno strumento di analisi nell'ambito della ricerca foscoliana, lo è altrettanto credere che la scelta di indossare delle maschere sia il punto d'arrivo della riflessione sul concetto di identità di cui si è parlato poc'anzi. Se così fosse, Foscolo starebbe affermando l'impossibilità di conoscersi come individui «indivisibili» e la conseguente necessità di accettare il proprio carattere pluriforme. Per quanto questa soluzione possa risultare affascinante rimane una speculazione arbitraria.

Non credo invece di commettere un errore riconoscendo una decisa diversificazione di funzioni per la prosa e la poesia nell'opera di Foscolo. Se la prosa è lo spazio della mistificazione, dello sperimentalismo di forme e contenuti, la poesia è il luogo della verità in cui è possibile depositare un autoritratto che seppur ritoccato più volte - si conferma uguale in tutte le sue versioni, dal 1808 al 1827.

\section{BIBLIOGRAFIA}

\section{Opere di Ugo Foscolo}

FoscOlo Ugo(1816), Ultime lettere, in : Id., Opere, II, Prose e saggi.

Foscolo Ugo (1831), Poesie inedite di Nicolò Ugo Foscolo tratte da un manoscritto originale, Lugano, Gius. Ruggia e C.

Foscolo Ugo (1837), Opere scelte di Ugo Foscolo, Parigi, Baudry, Libreria europea.

Foscolo Ugo (1842), Prose e poesie edite ed inedite di Ugo Foscolo ordinate da Luigi Carrer e corredate della vita dell'autore, Venezia, Co' Tipi del Gondoliere. 
Foscolo Ugo, (1853), Opere Edite e Postume di Ugo Foscolo, Firenze, Le Monnier.

FosColo Ugo (1890), Opere edite e postume di Ugo Foscolo (a cura di G. Chiarini), Firenze, Le Monnier.

FosColo Ugo (1904), Epistolario compreso quello amoroso di Ugo Foscolo e di Quirina Mocenni-Magiotti, Firenze, Salani.

FosCOLO Ugo (1904a), Poesie (a cura di G. Chiarini), Livorno, ed. Giusti.

FosCOLO Ugo (1933), Edizione Nazionale delle opere di Ugo Foscolo, vol. VII, Firenze, Le Monnier.

Foscolo Ugo (1933a), Didymi Clerici Prophetae minimi Hypercalypseos in Prose Politiche e Letterarie dal 1811 al 1816, E. N. vol. VIII, Firenze, Le Monnier, 1933, pp.65-118

FosCOLO Ugo (1933b), Lezioni, Articoli di critica e di polemica (1809-1811) (Edizione critica a cura di E. Santini.) E.N. vol. VII, Firenze, Le Monnier.

FosCOLO Ugo (1933c), Vestigi, in : Prose politiche e letterarie dal 1811 al 1816, E.N. vol. VIII, pp. 119-48, Firenze, Le Monnier.

FosColo Ugo (1933d), Poesie e carmi, E.N. vol. I, Firenze, Le Monnier.

Foscolo Ugo (1949), Epistolario di Ugo Foscolo volume I, (a cura di Plinio Carli), E.N. vol. XIV, Firenze, Le Monnier

Foscolo Ugo (1951), Prose varie d'arte, (a cura di Mario Fubini), E.N. vol. V, Firenze, Le Monnier

FosCOLO Ugo (1952), Epistolario di Ugo Foscolo volume II (a cura di Plinio Carli), E.N. vol. XV, Firenze, Le Monnier.

Foscolo Ugo (1953), Epistolario di Ugo Foscolo vol. III (a cura di Plinio Carli), E.N. XVI, Firenze, Le Monnier.

FosCOLO Ugo (1954), Epistolario di Ugo Foscolo, vol. IV (a cura di Plinio Carli), E.N. vol. XVII, Firenze, Le Monnier

FosColo Ugo (1956), Epistolario di Ugo Foscolo, vol.V, (a cura di Plinio Carli), E.N. vol. XVIII Firenze, Le Monnier.

FosCOLO Ugo (1961), Edizione Nazionale delle opere di Ugo Foscolo, vol. II, Firenze Le Monnier.

FosCOLO Ugo (1961a), Intendimento del traduttore, in : FosCOLO Ugo, E.N. vol. III, Firenze, Le Monnier, pp. 8-10.

FosColo Ugo (1966), Epistolario di Ugo Foscolo vol. VI, E.N. vol. XIX (a cura di Giovanni Gambarin e Francesco Tropeano), Firenze Le Monnier.

Foscolo Ugo (1970), Notizia Bibliografica in Ultime lettere di Jacopo Ortis, E.N. vol. IV, Firenze, Le Monnier, pp. 497-535.

FosCOlo Ugo (1970a), Epistolario di Ugo Foscolo vol. VII, (a cura di Mario Scotti), in : FosCOLO Ugo, E.N. vol. III, , Firenze, Le Monnier.

FosCOLO Ugo (1970b), Ultime lettere di Jacopo Ortis, E.N. vol. IV, Firenze, Le Monnier.

FosColo Ugo (1972), Scritti letterari e politici dal 1796 al 1808 (a cura di Giovanni Gambarin), E.N. vol.VI, Firenze, Le Monnier.

Foscolo Ugo (1974a), Le Grazie (a cura di Saverio Orlando), Brescia, Paideia Editrice. 
Foscolo Ugo (1974b), Epistolario, vol. VIII (1819-1821), (a cura di M. Scotti), E.N. vol. XVI, Firenze, Le Monnier.

FosCOLO Ugo (1974c), Le ultime lettere di Jacopo Ortis (a cura di Walter Binni e Lucio Felici), Milano, Garzanti.

Foscolo Ugo (1979), Ugo Foscolo, Storia della letteratura italiana, saggi raccolti e ordinati da Mario Alighiero Manacorda, Torino, Einaudi.

Foscolo Ugo (1980), Laurence Sterne Viaggio sentimentale, versione di Ugo Foscolo, Roma, Il Melograno.

Foscolo Ugo (1991), Sesto tomo dell'io (a cura di Vincenzo Di Benedetto), Torino, Einaudi.

FosCOLO Ugo (1992), Rime (a cura di Marco Cerruti), Milano, Feltrinelli.

FosCOLO Ugo (1994), Viaggio sentimentale di Laurence Sterne nella versione di Ugo Foscolo (a cura di Gianfranca Lavezzi), Milano, Rizzoli.

Foscolo Ugo (2005), Dell'origine e dell'ufficio della letteratura, Orazione, (a cura di Enzo Neppi), Leo S. Olschki Editore.

\section{Monografie critiche, antologie, studi particolari e su singole opere}

AMBrosino Paola (1989), La prosa epistolare del Foscolo, Firenze, La nuova Italia editrice.

ANTONELli Roberto ; SAPEGNO Maria Serena (2008), L'Europa degli scrittori, Il primo Ottocento: l'età del romanzo e il trionfo dell'Io, Milano, La Nuova Italia.

BARBARISI Gennaro (1987), Le ragioni della traduzione del "Viaggio Sentimentale", in : Atti dei convegni foscoliani, vol. III, Roma, Istituto Poligrafico e Zecca dello Stato Libreria di Stato.

CALVANI Alessandra (2004), Il viaggio italiano di Sterne, Firenze, Franco Cesati Editore.

De Robertis Giuseppe (1949), Primi studi manzoniani e altre cose, Firenze, Le Monnier.

DionisotTi CARLo (1988), Appunti sui Moderni, Foscolo, Leopardi, Manzoni e altri, Bologna, Il Mulino, pp. 33-53.

FUBINI MARIO (1963), Ortis e Didimo, Ricerche e interpretazioni foscoliane, Milano, Feltrinelli.

GoFFIS Cesare (1957), Ortis non scritto del 1814, in : Nuova Antologia, XCII 1957, pp. 53-84).

GoFFIS Cesare (1958), Nuovi studi foscoliani, Firenze, La Nuova Italia.

GRAF Arturo (1945), Rileggendo le «Ultime lettere di Jacopo Ortis», in : Id. Foscolo, Manzoni, Leopardi, Torino, Chiantore, pp. 1-22.

ISELLA Rita (1987a), La «fedeltà» nella prassi traduttoria del «Viaggio sentimentale», in : Atti dei convegni foscoliani, vol. III, Roma, Istituto Poligrafico e Zecca dello Stato Libreria di Stato. pp. 378-384

MASSANO Riccardo (1965), Goethe e Foscolo Werther e Ortis in Problemi di lingua e letteratura italiana del Settecento; Atti del quarto congresso dell'associazione internazionale per gli studi di lingua e letteratura italiana, Wiesbaden, Franz Steiner Verlang Gmbh. 
MAZZACURATI Giancarlo (1990), Effetto Sterne: la narrazione umoristica in Italia da Foscolo a Pirandello, Pisa, Nistri - Lischi.

MorABITO Raffaele (1977), Antiromanzi dell'Ottocento, Foscolo-Sterne, Tommaseo, Verga, Oriani, D’Annunzio, Roma, Bulzoni editore.

NiCOLETTI Giuseppe (1978), Il «metodo» dell'«Ortis»e altri studi foscoliani, Firenze, La Nuova Italia Editrice.

Palumbo Matteo (1990a), Jacopo Ortis, Didimo Chierico e gli avvertimenti di Foscolo «Al lettore», in : Effetto Sterne, La Narrazione umoristica in Italia da Foscolo a Pirandello, Nistri-Lischi, Pisa, 1990, pp. 60-90.

RABIZZANI Giovanni (1920), Sterne in Italia: riflessi nostrani dell'umorismo sentimentale; con prefazione di Odiardo Gori, Roma, Formiggini.

SoFFICI Ardengo (1922), Le più belle pagine di Ugo Foscolo scelte da Ardengo Soffici, Milano, fratelli Treves editori.

TERzOLI Maria Antonietta (2007), Con l'incantesimo della parola, Foscolo scrittore e critico, Roma, Edizioni di Storia e Letteratura.

TOSCHI Luca (1987b), Foscolo lettore di Sterne e altri «Sentimental Travellers» in : Atti dei convegni foscoliani, vol. III, Roma, Istituto Poligrafico e Zecca dello Stato Libreria di Stato. pp. 480-495.

TrischitTA Giovanni (1905), Storia ed estetica delle Ultime lettere di Jacopo Ortis di Niccolò Ugo Foscolo, Messina, Vincenzo Muglia Editore.

UNIVERsità DI PAVIA (1927) (a cura di), Studi su Ugo Foscolo (1927), Torino, Casa Editrice Giovanni Chiantore.

VARESE Claudio (1947), Linguaggio sterniano e linguaggio foscoliano, Firenze, Sansoni.

\section{Letteratura secondaria sul genere autobiografico}

ACCETTO Torquato (1943), Della dissimulazione onesta, a cura di Goffredo Bellonci, Firenze, Le Monnier, 1943.

BARTOLO Anglani (a cura di) (1996), Teorie moderne dell'autobiografia, Bari, B. A. Graphis, 1996.

BATtistini Andrea (1990), Lo specchio di Dedalo : autobiografia e biografia, Bologna, Il mulino.

CARBone Paola (2008), La lanterna magica di Tristram Shandy : visualità e informazione, ordine ed entropia, paradossi e trompe-l'oeil nel romanzo di Laurence Sterne, Verona, Ombre corte.

D'InTINO Franco (1998), L'autobiografia moderna : storia, forme, problemi, Roma, Bulzoni.

GENTILI (1997) = Sandro G., I codici autobiografici di Ugo Foscolo, Roma, Bulzoni Editore.

JONARD Norbert (1987), Le «Ultime lettere di Jacopo Ortis» ed i problemi dell'autobiografia romanzesca in : «Atti dei convegni foscoliani», vol. I, Roma, Istituto Poligrafico e Zecca dello Stato, Libreria di Stato. pp. 327-351.

MORABITO Raffaele (1977), Antiromanzi dell'Ottocento: Foscolo-Sterne, Tommaseo, Verga, Oriani, D’Annunzio, Roma : Bulzoni.

\section{Sitografia}


Foscolo (1993), Le ultime lettere di Jacopo Ortis, Roma Newton Compton, versione digitale in

http://www.letteraturaitaliana.net/pdf/Volume_8/t167.pdf, consultato il 2.2.2017 FosCOLO Ugo (2008), Carteggio con Antonietta Fagnani Arese, in : Foscolo in Epistolario 1794-1804, versione digitale in www.bibliotecaitaliana.it consultato il 2.2.2017 